\title{
How the neck affects the back: changes in regional cervical sagittal alignment correlate to HRQOL improvement in adult thoracolumbar deformity patients at 2-year follow-up
}

\author{
Themistocles S. Protopsaltis, MD, ${ }^{1}$ Justin K. Scheer, BS, ${ }^{2}$ Jamie S. Terran, BS, ${ }^{1}$ \\ Justin S. Smith, MD, PhD, ${ }^{3}$ D. Kojo Hamilton, MD, ${ }^{4}$ Han Jo Kim, MD, ${ }^{5}$ Greg M. Mundis Jr., MD, ${ }^{6}$ \\ Robert A. Hart, MD, ${ }^{4}$ Ian M. McCarthy, PhD, ${ }^{7}$ Eric Klineberg, MD, ${ }^{8}$ Virginie Lafage, PhD, ${ }^{1}$ \\ Shay Bess, MD, ${ }^{9}$ Frank Schwab, MD, ${ }^{1}$ Christopher I. Shaffrey, MD, ${ }^{3}$ Christopher P. Ames, MD, ${ }^{10}$ \\ and the International Spine Study Group
}

\begin{abstract}
1Department of Orthopaedic Surgery, NYU Hospital for Joint Diseases, New York, New York; ${ }^{2}$ Department of Neurological Surgery, Northwestern University Feinberg School of Medicine, Chicago, Illinois; ${ }^{3}$ Department of Neurosurgery, University of Virginia Health System, Charlottesville, Virginia; ${ }^{\circ}$ Department of Orthopaedic Surgery, Oregon Health \& Science University, Portland, Oregon; ${ }^{5}$ Department of Orthopaedic Surgery, Hospital for Special Surgery, New York, New York; ${ }^{6}$ San Diego Center for Spinal Disorders, La Jolla, California; ' $D$ Department of Orthopaedic Surgery, Baylor Scoliosis Center, Plano, Texas; ${ }^{8}$ Department of Orthopaedic Surgery, University of California, Davis, California; ${ }^{9}$ Rocky Mountain Hospital for Children, Denver, Colorado; and ${ }^{10}$ Department of Neurological Surgery, University of California, San Francisco, California
\end{abstract}

OBJECT Regional cervical sagittal alignment (C2-7 sagittal vertical axis [SVA]) has been shown to correlate with health-related quality of life (HRQOL). The study objective was to examine the relationship between cervical and thoracolumbar alignment parameters with $\mathrm{HRQOL}$ among patients with operative and nonoperative adult thoracolumbar deformity.

METHODS This is a multicenter prospective data collection of consecutive patients with adult thoracolumbar spinal deformity. Clinical measures of disability included the Oswestry Disability Index (ODI), Scoliosis Research Society-22 Patient Questionnaire (SRS-22), and 36-Item Short-Form Health Survey (SF-36). Cervical radiographic parameters were correlated with global sagittal parameters within the nonoperative and operative cohorts. A partial correlation analysis was performed controlling for C-7 SVA. The operative group was subanalyzed by the magnitude of global deformity (C-7 $S V A \geq 5 \mathrm{~cm}$ vs $<5 \mathrm{~cm}$ ).

RESULTS A total of 318 patients were included (186 operative and 132 nonoperative). The mean age was $55.4 \pm 14.9$ years. Operative patients had significantly worse baseline HRQOL and significantly larger C-7 SVA, pelvic tilt (PT), mismatch between pelvic incidence and lumbar lordosis (PI-LL), and C2-7 SVA. The operative patients with baseline C-7

\footnotetext{
ABBREVIATIONS BMI = body mass index; $\mathrm{CCl}=$ Charlson Comorbidity Index; $\mathrm{CL}=\mathrm{C2}-7$ lordosis; $\mathrm{HRQOL}=$ health-related quality of life; ISSG = International Spine Study Group; LL = lumbar lordosis; MCS = Mental Component Summary; NDI = Neck Disability Index; NRS = numeric rating scale; ODI = Oswestry Disability Index; PCS = Physical Component Summary; PI-LL = mismatch between pelvic incidence and LL; PT = pelvic tilt; SF-36 = 36-Item Short-Form Health Survey; SRS-22 = Scoliosis Research Society-22 Patient Questionnaire; SVA = sagittal vertical axis; TK = T2-12 kyphosis; T1S = T-1 slope; T1S-CL = T1S minus CL.

SUBMITTED January 11, 2014. ACCEPTED November 25, 2014.

INCLUDE WHEN CITING Published online May 15, 2015; DOI: 10.3171/2014.11.SPINE1441.

DISCLOSURE The authors report the following. Dr. Protopsaltis: consultant for Globus; Speaker's Bureau for K2M, Alphatec. Dr. Smith: consultant for Biomet, DePuy, Globus, Medtronic; support for study described and non-study-related clinical/research effort from DePuy/ISSGF. Dr. Mundis: consultant for NuVasive, K2M; support for nonstudy-related clinical/research effort from ISSGF, NuVasive. Dr. Hart: consultant for DePuy, Medtronic; direct stock ownership in Spine Connect; patent holder with OHSU; non-study-related clinical/research effort from DePuy/ISSGF; expert testimonies for Evans, Craven, \& Lackie and Benson, Bertoldo, Baker, \& Carter. Dr. Klineberg: speaker fees from DePuy, AO Spine, Stryker; grants for fellowship from OREF, DePuy; research grant from AO Spine. Dr. Bess: consultant for K2M, DePuy Spine, Allosource; support for study described from DePuy; support for non-study-related clinical/research effort from Medtronic. Dr. Shaffrey: consultant for Biomet, Globus, Medtronic, NuVasive, Stryker; patent holder with and receives royalties from Biomet, Medtronic. Dr. Ames: consultant for DePuy, Medtronic, Stryker; direct stock ownership in Doctors Research Group, Visualase, Baxano Surgery; patent holder with Fish \& Richardson, P.C.; receives royalties from Aesculap, Biomet Spine. The ISSG is funded through research grants from DePuy Spine and individual donations.
} 
SVA $\geq 5 \mathrm{~cm}$ had significantly larger C2-7 lordosis (CL), C2-7 SVA, C-7 SVA, PI-LL, and PT than patients with a normal C-7 SVA. For all patients, baseline C2-7 SVA and CL significantly correlated with baseline ODI, Physical Component Summary (PCS), SRS Activity domain, and SRS Appearance domain. Baseline C2-7 SVA also correlated with SRS Pain and SRS Total. For the operative patients with baseline C-7 SVA $\geq 5 \mathrm{~cm}$, the 2-year C2-7 SVA significantly correlated with 2-year Mental Component Summary, SRS Mental, SRS Satisfaction, and decreases in ODI. Decreases in C2-7 SVA at 2 years significantly correlated with lower ODI at 2 years. Using partial correlations while controlling for C-7 SVA, the C2-7 SVA correlated significantly with baseline ODI $(r=0.211, p=0.002)$, PCS $(r=-0.178, p=0.009)$, and SRS Activity $(r=-0.145, p=0.034)$ for the entire cohort. In the subset of operative patients with larger thoracolumbar deformities, the change in C2-7 SVA correlated with change in ODI ( $r=-0.311, p=0.03$ ).

CONCLUSIONS Changes in cervical lordosis correlate to HRQOL improvements in thoracolumbar deformity patients at 2-year follow-up. Regional cervical sagittal parameters such as CL and C2-7 SVA are correlated with clinical measures of regional disability and health status in patients with adult thoracolumbar scoliosis. This effect may be direct or a reciprocal effect of the underlying global deformities on regional cervical alignment. However, the partial correlation analysis, controlling for the magnitude of the thoracolumbar deformity, suggests that there is a direct effect of cervical alignment on health measures. Improvements in regional cervical alignment postoperatively correlated positively with improved HRQOL.

http://thejns.org/doi/abs/10.3171/2014.11.SPINE1441

KEY WORDS adult; cervical deformity; cervical lordosis; outcomes; sagittal alignment; spine; surgery

$\mathrm{A}$ DULT spinal deformity has been studied extensively in the literature with a majority of publications focusing on thoracolumbar deformity and its effect on health-related quality of life (HRQOL) measures. ${ }^{13,6,7,10,11,13,14,16,17}$ Among patients with thoracolumbar deformities, positive sagittal alignment has been associated with pain and disability., ${ }^{2,11,13,16}$ Few studies have correlated validated health measures and positive cervical sagittal alignment. ${ }^{16,17}$ Tang et al. ${ }^{16}$ demonstrated that a C2-7 sagittal vertical axis (SVA) greater than $4 \mathrm{~cm}$ correlated with increased disability in patients following posterior cervical fusion procedures. Smith and colleagues demonstrated that thoracolumbar sagittal deformities cause a compensatory reciprocal cervical hyperlordosis to maintain horizontal gaze. ${ }^{15}$ This reciprocal change was shown to reverse with postoperative deformity correction. ${ }^{15}$

However, the effect of cervical sagittal alignment on HRQOL in patients with thoracolumbar sagittal deformities has not been investigated extensively. In the present study we hypothesize that cervical sagittal malalignment will negatively impact health measures in patients with thoracolumbar deformities and improvement in cervical sagittal alignment after thoracolumbar deformity correction will have a corresponding improvement in HRQOL.

\section{Methods \\ Patient Population}

This is a multicenter prospective data collection of consecutive patients with adult thoracolumbar spinal deformity conducted through the International Spine Study Group (ISSG), which is composed of 11 sites across the United States. Patients were enrolled at each site through an institutional review board-approved protocol. Inclusion criteria for the ISSG database are age older than 18 years and presence of at least one of the following measures of spinal deformity: scoliosis Cobb angle $\geq 20^{\circ}, \mathrm{SVA} \geq 5 \mathrm{~cm}$, pelvic tilt $(\mathrm{PT}) \geq 25^{\circ}$, and thoracic kyphosis $(\mathrm{TK}) \geq 60^{\circ}$. Patients were divided into nonoperative and operative cohorts. The operative group was subanalyzed by the magnitude of global deformity (SVA $\geq 5 \mathrm{~cm}$ vs $\mathrm{SVA}<5 \mathrm{~cm}$ ).

\section{Data Collection and Radiographic Assessment}

Demographic, clinical, radiographic, and HRQOL data collection was performed at baseline and at the 2-year follow-up. The demographic and clinical data included patient age, sex, body mass index (BMI), and Charlson Comorbidity Index (CCI). ${ }^{5} \mathrm{~A}$ basic numeric rating scale (NRS) score ranging from 0 (no pain) to 10 (most unbearable pain) was collected for back and leg pain.

Standardized HRQOL measures included the Oswestry Disability Index (ODI), 36-Item Short-Form Health Survey (SF-36), and Scoliosis Research Society-22 Patient Questionnaire (SRS-22). Two standard summary scores were calculated based on the SF-36: the Physical Component Summary (PCS) and the Mental Component Summary (MCS) scores. The SRS-22 provides a total score and multiple subdomains, including Activity, Pain, Appearance, Mental, and Satisfaction.

Full-length 36-in free-standing anteroposterior and lateral spine radiographs were obtained at baseline and 1- and 2-year follow-up. Radiographic analysis was performed at 1 site (New York University) with extensive experience using validated software (Spineview, ENSAM). ${ }^{4}$ Cervical radiographic measures included C2-7 SVA (C-2 plumb line relative to C-7), C2-7 lordosis (CL), T-1 slope, and T-1 slope minus CL (T1S-CL). Thoracolumbar radiographic measures included T2-12 kyphosis (TK, Cobb angle between superior endplate of T-2 and inferior endplate of T-12), lumbar lordosis (LL, Cobb angle between inferior endplate of L-1 and superior endplate of S-1), C-7 SVA (C-7 plumb line relative to S-1), PT, and mismatch between pelvic incidence and lumbar lordosis (PI-LL). 


\section{Data and Statistical Analysis}

Mean and standard deviation were used to describe continuous variables, and frequency analyses were used for categorical variables. Demographic, clinical, radiographic, and HRQOL measures were compared at baseline and 2-year follow-up using the paired t-test, unpaired t-test, and Wilcoxon rank-sum test where appropriate. Patients were divided into nonoperative and operative cohorts. The operative group was subanalyzed by the magnitude of global deformity (SVA $\geq 5 \mathrm{~cm}$ vs SVA < $5 \mathrm{~cm})$. A multivariate regression analysis controlling for thoracolumbar deformity (C-7 SVA) was performed to identify the cervical parameters that are predictors of poor HRQOL. Partial correlations were performed controlling for the SRS Schwab thoracolumbar deformity modifiers. The level of significance for all statistical tests was set at $\mathrm{p}<0.05$.

\section{Results}

\section{Patient Populations, Demographics, and Comparison of Baseline Values}

A total of 318 patients were included (186 operative and 132 nonoperative; Table 1). The overall mean age was $55.4 \pm 14.9$ years. The operative patients had a higher BMI and CCI than the nonoperative patients $(\mathrm{p}<0.05$ for both; Table 1). Furthermore, within the operative patients, the patients with larger C-7 SVA $(\geq 5 \mathrm{~cm})$ were significantly older and had higher BMI and CCI than the patients with normal C-7 SVA $(<5 \mathrm{~cm})(\mathrm{p}<0.05$ for all; Table 1). The operative patients had significantly worse baseline HRQOL in all parameters measured than the nonoperative patients ( $\mathrm{p}<0.05$ for all; Table 2$)$. Similarly within the operative patients, the patients with larger baseline C-7 SVA had significantly worse baseline HRQOL in all parameters measured than the patients with normal baseline C-7 SVA $(p<0.05$; Table 2) with the exception of the SRS Satisfaction domain. Baseline LL for the nonoperative patients was significantly larger than for the operative patients ( $\mathrm{p}<0.05$; Table 3). C-7 SVA, PT, PI-LL, and C2-7 SVA were significantly higher in the operative patients compared with the nonoperative patients $(\mathrm{p}<0.05$ for all; Table 3). There were no significant differences between the operative and nonoperative groups regarding baseline $\mathrm{CL}$ and T1S-CL ( $p>0.05$ for all). The operative patients with baseline C-7 SVA $\geq 5 \mathrm{~cm}$ had significantly greater T1S, CL, C-7 SVA, PI-LL, C2-7 SVA, and PT and sig- nificantly smaller LL than the patients with a normal C-7 SVA ( $p<0.05$ for all; Table 3).

\section{Multivariate Regression Analysis}

At baseline, a multivariate regression analysis controlling for thoracolumbar deformity (C-7 SVA) demonstrated that C2-7 SVA $(p<0.001)$ and T1S-CL $(p=0.02)$ were independent predictors of disability as measured by ODI. The regression analysis produced an $r$ value of 0.470 , with a $\mathrm{p}$ value of 0.002 .

\section{Correlations of Cervical Radiographic Parameters and HRQOL}

For all patients, both baseline C2-7 SVA and baseline CL significantly correlated with baseline ODI, PCS, SRS Activity, and SRS Appearance ( $\mathrm{p}<0.05$ for all; Table 4). Baseline C2-7 SVA also correlated with SRS Pain and SRS Total $(\mathrm{p}<0.05$ for all; Table 4). For the operative patients, 2-year C2-7 SVA and CL significantly correlated with 2-year PCS and SRS Activity, respectively $(\mathrm{p}<0.05$ for both; Table 5). Two-year T1S significantly correlated with 2-year ODI, PCS, SRS Activity, SRS Appearance, and SRS Total ( $\mathrm{p}<0.05$ for all; Table 5).

Using partial correlations on the entire patient cohort at baseline while controlling for C-7 SVA, the C2-7 SVA correlated significantly with baseline ODI $(r=0.211, p=$ $0.002)$, PCS $(r=-0.178, p=0.009)$, and SRS Activity $(r=$ $-0.145, \mathrm{p}=0.034)$.

\section{Correlations for Operative Patients With Baseline C-7 SVA $\geq 5 \mathrm{~cm}$}

The baseline C2-7 SVA significantly correlated with baseline T1S-CL, T1S, TK, and CL ( $p<0.05$ for all; Table 6). Baseline CL significantly correlated with baseline TK and baseline T1S ( $p<0.05$ for both; Table 6). Changes from baseline to 2 years for C2-7 SVA correlated with change in T1S-CL and T1S ( $<0.05$ for both; Table 6). Change in CL correlated with change in T1S and T1S-CL ( $p<0.05$ for both; Table 6). Two-year C2-7 SVA significantly correlated with 2-year MCS, SRS Mental, SRS Satisfaction, and decreases in ODI ( $1<0.05$ for all; Table 7). Decreases in C2-7 SVA at 2 years significantly correlated with lower ODI at 2 years $(\mathrm{p}<0.05$ for all; Table 7$)$.

Using partial correlations while controlling for change in C-7 SVA in patients with larger thoracolumbar deformities (baseline $\mathrm{C}-7 \mathrm{SVA} \geq 5 \mathrm{~cm}$ ), the change in $\mathrm{C} 2-7$

TABLE 1. Baseline demographics by treatment modality and by baseline C-7 SVA*

\begin{tabular}{|c|c|c|c|c|c|c|}
\hline \multirow[b]{2}{*}{ Variable } & \multicolumn{3}{|c|}{ Comparison by Treatment Modality } & \multicolumn{3}{|c|}{ Comparison by Baseline C-7 SVA (operative patients only) } \\
\hline & Operative & Nonoperative & p Value & C-7 SVA $\geq 5 \mathrm{~cm}$ & C-7 SVA $<5 \mathrm{~cm}$ & $p$ Value \\
\hline No. of patients & 186 & 132 & & 88 & 98 & \\
\hline Female/male & $160: 26$ & $114: 18$ & & $68: 20$ & $92: 6$ & \\
\hline Mean age in yrs & $55.9 \pm 14.8$ & $54.6 \pm 15$ & NS & $63.8 \pm 11.7$ & $49.5 \pm 16.2$ & $<0.0001$ \\
\hline Mean BMI & $27.6 \pm 6.8$ & $25.7 \pm 6.2$ & $<0.0001$ & $29.2 \pm 6.5$ & $25.8 \pm 6.4$ & $<0.0001$ \\
\hline Mean $\mathrm{CCl}$ & $1.5 \pm 1.6$ & $1.1 \pm 1.4$ & 0.0002 & $1.9 \pm 1.8$ & $1.1 \pm 1.4$ & 0.0001 \\
\hline
\end{tabular}


TABLE 2. Baseline HRQOL values by treatment modality and by baseline C-7 SVA*

\begin{tabular}{|c|c|c|c|c|c|c|}
\hline \multirow[b]{2}{*}{ Variable } & \multicolumn{3}{|c|}{ Comparison by Treatment Modality } & \multicolumn{3}{|c|}{ Comparison by Baseline C-7 SVA (operative patients only) } \\
\hline & Operative & Nonoperative & p Value & C-7 SVA $\geq 5 \mathrm{~cm}$ & C-7 SVA $<5 \mathrm{~cm}$ & $p$ Value \\
\hline Back pain NRS & $7.1 \pm 2.3$ & $4.6 \pm 2.6$ & $<0.0001$ & $7.6 \pm 2.0$ & $6.5 \pm 2.5$ & $<0.0001$ \\
\hline Leg pain NRS & $4.3 \pm 3.3$ & $2.6 \pm 3$ & $<0.0001$ & $4.8 \pm 3.2$ & $3.9 \pm 3.5$ & $<0.0001$ \\
\hline ODI & $41.3 \pm 18.9$ & $24.1 \pm 16.1$ & $<0.0001$ & $47.1 \pm 16.2$ & $36.1 \pm 19.8$ & $<0.0001$ \\
\hline PCS & $32.8 \pm 9.7$ & $42.8 \pm 10.1$ & $<0.0001$ & $28.8 \pm 8.7$ & $36.5 \pm 9.1$ & $<0.0001$ \\
\hline MCS & $46.1 \pm 13.1$ & $50.8 \pm 11$ & 0.0017 & $44.6 \pm 13.3$ & $47.4 \pm 12.8$ & 0.0031 \\
\hline SRS Activity & $3.0 \pm 0.9$ & $3.9 \pm 0.8$ & $<0.0001$ & $2.7 \pm 0.8$ & $3.3 \pm 0.9$ & $<0.0001$ \\
\hline SRS Pain & $2.5 \pm 0.8$ & $3.3 \pm 0.9$ & $<0.0001$ & $2.3 \pm 0.7$ & $2.8 \pm 0.9$ & $<0.0001$ \\
\hline SRS Appearance & $2.5 \pm 0.8$ & $3.3 \pm 0.7$ & $<0.0001$ & $2.3 \pm 0.8$ & $2.7 \pm 0.7$ & $<0.0001$ \\
\hline SRS Mental & $3.5 \pm 0.9$ & $3.9 \pm 0.8$ & 0.0002 & $3.4 \pm 1.0$ & $3.6 \pm 0.8$ & 0.0011 \\
\hline SRS Satisfaction & $2.7 \pm 1.0$ & $3.3 \pm 1.1$ & $<0.0001$ & $2.6 \pm 1.0$ & $2.9 \pm 1.0$ & $>0.05$ \\
\hline SRS Total & $2.9 \pm 0.7$ & $3.6 \pm 0.6$ & $<0.0001$ & $2.7 \pm 0.6$ & $3.1 \pm 0.6$ & $<0.0001$ \\
\hline
\end{tabular}

* Mean values are presented as the mean \pm SD.

SVA correlated significantly with the change in ODI $(r=$ $-0.311, \mathrm{p}=0.03$ ).

\section{Discussion}

It has been well established that patients with thoracolumbar deformity and positive sagittal alignment have increased pain and disability. 2,7,10,11,13 Few studies exist to correlate cervical sagittal alignment with validated HRQOL. ${ }^{16,17}$ Tang and colleagues demonstrated that among 113 patients undergoing posterior cervical fusion procedures, positive cervical sagittal alignment had a detrimental effect on validated health measures including the SF-36 and Neck Disability Index (NDI). ${ }^{16}$ Villavicencio et al. conducted a prospective randomized controlled trial of lordotic and parallel interbody grafts for anterior cervical discectomy and fusion in 122 patients to assess the effect of cervical sagittal alignment on HRQOL. ${ }^{17}$ They showed that patients whose cervical sagittal alignment was maintained or improved had significantly greater improvement in SF-36 and NDI outcomes. ${ }^{17}$

While changes in reciprocal regional spinal alignment have been demonstrated after thoracolumbar deformity correction, the effect of these changes on HRQOL has not been well established. ${ }^{15}$ Lafage et al. ${ }^{9}$ demonstrated that a reciprocal increase in thoracic kyphosis occurs after lumbar pedicle subtraction osteotomy, whereas Klineberg et al. ${ }^{8}$ demonstrated that thoracic osteotomies for correction of kyphosis resulted in a reciprocal decrease in lumbar lordosis. Smith and colleagues studied 75 patients with thoracolumbar deformity and positive sagittal alignment and showed that reciprocal cervical hyperlodosis results in an effort to maintain horizontal gaze. ${ }^{15}$ This reciprocal cervical hyperlordosis corrects after pedicle subtraction osteotomy and corrections of the underlying thoracolumbar malaligment. ${ }^{15}$

This study demonstrates that baseline cervical sagittal malalignment has a detrimental effect on validated health measures. Regional cervical sagittal parameters, such as cervical lordosis and C2-7 SVA, were correlated with clinical measures of disability and health status in patients with adult thoracolumbar scoliosis. Patients with greater global deformities (SVA $\geq 5 \mathrm{~cm}$ ) had larger baseline cervical lordosis, T1S, and C2-7 SVA and lower HRQOL than those with SVA $<5 \mathrm{~cm}$. Moreover, following deformity correction, improvements in regional cervical alignment resulted in improvements in HRQOL. A decrease in C2-7 SVA correlated with HRQOL at 2 years in patients with SVA $\geq 5 \mathrm{~cm}$. For this group, the 2-year C2-7 SVA correlated with 2-year MCS, SRS Mental, and SRS Satisfaction. Improvements in the C2-7 SVA correlated with improvements in ODI at 2 years. It is difficult to determine if the improvements in HRQOL were a result of the reciprocal cervical alignment correction or the global deformity realignment. However, it is our experience that patients will often have dual complaints of low-back pain and neck pain, with both complaints improving following global spinal deformity correction. Nevertheless, even

TABLE 3. Baseline radiographic parameters by treatment modality and by baseline C-7 SVA*

\begin{tabular}{|c|c|c|c|c|c|c|}
\hline \multirow[b]{2}{*}{ Variable } & \multicolumn{3}{|c|}{ Comparison by Treatment Modality } & \multicolumn{3}{|c|}{ Comparison by Baseline C-7 SVA } \\
\hline & Operative & Nonoperative & p Value & C-7 SVA $\geq 5 \mathrm{~cm}$ & C-7 SVA $<5 \mathrm{~cm}$ & $p$ Value \\
\hline C2-7 lordosis & $8.1 \pm 15.3$ & $5.6 \pm 13.2$ & $>0.05$ & $13.5 \pm 14.5$ & $3.5 \pm 14.6$ & $<0.001$ \\
\hline C2-7 SVA (cm) & $3.6 \pm 1.7$ & $2.9 \pm 1.6$ & 0.0035 & $4.0 \pm 1.8$ & $3.2 \pm 1.6$ & 0.0003 \\
\hline LL & $37.1 \pm 23.1$ & $47.2 \pm 17.8$ & $<0.0001$ & $26 \pm 22.5$ & $47.0 \pm 18.8$ & $<0.001$ \\
\hline PT & $23.8 \pm 11.4$ & $20.3 \pm 9.9$ & 0.0035 & $29.7 \pm 11.5$ & $18.5 \pm 8.4$ & $<0.001$ \\
\hline C-7 SVA (cm) & $5.6 \pm 7.7$ & $2.6 \pm 5.3$ & 0.0018 & $12.3 \pm 5.9$ & $-0.1 \pm 3.1$ & $<0.001$ \\
\hline PI-LL & $14.2 \pm 21.5$ & $6.2 \pm 16.1$ & 0.0010 & $2.7 \pm 2.0$ & $0.2 \pm 1.5$ & $<0.001$ \\
\hline
\end{tabular}

* Mean values are presented as the mean \pm SD. 
TABLE 4. Baseline correlations for all patients $(n=318)$ regarding C2-7 SVA and CL with HRQOL

\begin{tabular}{clrr}
\hline $\begin{array}{c}\text { Baseline Alignment } \\
\text { Measurement }\end{array}$ & \multicolumn{1}{c}{$\begin{array}{c}\text { Baseline Outcome } \\
\text { Measure }\end{array}$} & r Value & $p$ Value \\
\hline C2-7 SVA & ODI & 0.26 & $<0.0001$ \\
\hline C2-7 SVA & PCS & -0.26 & $<0.0001$ \\
\hline C2-7 SVA & SRS Activity & -0.19 & 0.0038 \\
\hline C2-7 SVA & SRS Pain & -0.18 & 0.0061 \\
\hline C2-7 SVA & SRS Appearance & -0.18 & $<0.0001$ \\
\hline C2-7 SVA & SRS Total & -0.17 & $<0.0001$ \\
\hline CL & ODI & 0.17 & 0.0097 \\
\hline CL & PCS & -0.18 & 0.0072 \\
\hline CL & SRS Activity & -0.13 & 0.0439 \\
\hline CL & SRS Appearance & -0.15 & 0.0120 \\
\hline
\end{tabular}

when controlling for the magnitude of the thoracolumbar deformity, the multivariate regression analysis showed that the C2-7 SVA parameter plays a role in HRQOL. Moreover, the partial correlation analysis controlling for C-7 SVA showed that in the operative cohort with the larger thoracolumbar deformities, the C2-7 SVA correlated with many of the HRQOL measures. These analyses suggest that the C2-7 parameter in particular affects health status and disability even in the setting of population with primary thoracolumbar deformity.

While general health status measures such as the SF-36 were employed, and disease-specific questionnaires such as the SRS-22 and the low-back pain-specific ODI were used, a primary limitation of the study is that a neck painspecific questionnaire such as the NDI was not employed. However, it is important to note that a questionnaire specific to cervical sagittal deformity does not exist. In an investigation correlating ODI and NDI scores in patients presenting with primary cervical, primary lumbar, and combined complaints, the ODI scores were elevated in patients with primary complaints related to the cervical spine. ${ }^{12}$ The converse was true for patients with primary lumbar complaints. It was postulated that this effect results from the fact that the NDI was developed as a modification of the ODI with many of the questions repeated in the 2 questionnaires. Therefore, while the use of the NDI would have strengthened the aims of this study, the general health status measures and the ODI are useful instruments in assessing disability related to cervical malalignment. Another limitation of this study is the retrospective design, although the analyses were based on prospectively collected data. The strengths of the study include the contribution of cases from multiple spinal deformity centers and the use of multiple standardized measures of HRQOL with 2-year follow-up.

TABLE 5. Correlations of C2-7 SVA and CL with HRQOL for operative patients at 2 years

\begin{tabular}{cccc}
\hline 2-Yr Measurement & 2-Yr Outcome Measure & r Value & $p$ Value \\
\hline C2-7 SVA & PCS & -0.17 & 0.0267 \\
\hline CL & SRS Activity & -0.18 & 0.0173 \\
\hline
\end{tabular}

TABLE 6. Baseline and 2-year radiographic correlations for operative patients with global sagittal malalignment (C-7 SVA $\geq$ $5 \mathrm{~cm}$ )

\begin{tabular}{llrr}
\hline \multicolumn{2}{c}{ Alignment Measure Correlations } & r Value & $p$ Value \\
\hline Baseline C2-7 SVA & Baseline T1S-CL & 0.51 & $<0.0001$ \\
\hline Baseline C2-7 SVA & Baseline T1S & 0.51 & $<0.0001$ \\
\hline Baseline C2-7 SVA & Baseline TK & -0.32 & 0.0071 \\
\hline Baseline C2-7 SVA & Baseline CL & 0.31 & 0.0104 \\
\hline Baseline C2-7 SVA & Baseline PI-LL & 0.54 & $<0.0001$ \\
\hline Baseline CL & Baseline TK & -0.38 & $<0.0001$ \\
\hline Baseline CL & Baseline T1S & 0.68 & 0.0011 \\
\hline 2-yr C2-7 SVA change & 2-yr T1S change & 0.44 & 0.0003 \\
\hline 2-yr C2-7 SVA change & 2-yr T1S-CL change & 0.52 & $<0.0001$ \\
\hline 2-yr CL change & 2-yr T1S change & 0.47 & 0.0001 \\
\hline 2-yr CL change & 2-yr T1S-CL change & 0.66 & $<0.0001$ \\
\hline
\end{tabular}

Change $=$ difference between the listed postoperative time point and the preoperative value.

TABLE 7. Correlations of operative patients with global sagittal malalignment (C-7 SVA $\geq 5 \mathrm{~cm}$ ) for 2-year C2-7 SVA and HRQOL

\begin{tabular}{llrl}
\hline \multicolumn{1}{c}{ Alignment Measure } & \multicolumn{1}{c}{ Outcome Measure } & r Value & $p$ Value \\
\hline 2-yr C2-7 SVA & 2-yr MCS & 0.24 & 0.0350 \\
\hline 2-yr C2-7 SVA & 2-yr SRS Mental & 0.29 & 0.0100 \\
\hline 2-yr C2-7 SVA & 2-yr SRS Satisfaction & 0.23 & 0.0408 \\
\hline 2-yr C2-7 SVA & 2-yr ODI change & -0.22 & 0.0456 \\
\hline 2-yr C2-7 SVA change & 2-yr ODI & -0.31 & 0.0123 \\
\hline 2-yr C2-7 SVA change & 2-yr ODI change & -0.30 & 0.0179 \\
\hline
\end{tabular}

Change $=$ difference between the listed postoperative time point and the preoperative value.

\section{Conclusions}

This study demonstrates that following thoracolumbar deformity correction, there is a direct, albeit smaller, beneficial effect on health measures of improved cervical sagittal alignment and reduced cervical hyperlordosis for maintenance horizontal gaze.

\section{References}

1. Ames CP, Smith JS, Scheer JK, Bess S, Bederman SS, Deviren $\mathrm{V}$, et al: Impact of spinopelvic alignment on decision making in deformity surgery in adults. A review. J Neurosurg Spine 16:547-564, 2012

2. Bridwell KH, Baldus C, Berven S, Edwards C II, Glassman $\mathrm{S}$, Hamill C, et al: Changes in radiographic and clinical outcomes with primary treatment adult spinal deformity surgeries from two years to three- to five-years follow-up. Spine (Phila Pa 1976) 35:1849-1854, 2010

3. Bridwell KH, Berven S, Glassman S, Hamill C, Horton WC III, Lenke LG, et al: Is the SRS-22 instrument responsive to change in adult scoliosis patients having primary spinal deformity surgery? Spine (Phila Pa 1976) 32:2220-2225, 2007

4. Champain S, Benchikh K, Nogier A, Mazel C, Guise JD, Skalli W: Validation of new clinical quantitative analysis 
software applicable in spine orthopaedic studies. Eur Spine J 15:982-991, 2006

5. Charlson M, Szatrowski TP, Peterson J, Gold J: Validation of a combined comorbidity index. J Clin Epidemiol 47:12451251,1994

6. Glassman SD, Berven S, Bridwell K, Horton W, Dimar JR: Correlation of radiographic parameters and clinical symptoms in adult scoliosis. Spine (Phila Pa 1976) 30:682-688, 2005

7. Glassman SD, Bridwell K, Dimar JR, Horton W, Berven S, Schwab F: The impact of positive sagittal balance in adult spinal deformity. Spine (Phila Pa 1976) 30:2024-2029, 2005

8. Klineberg E, Schwab F, Ames C, Hostin R, Bess S, Smith JS, et al: Acute reciprocal changes distant from the site of spinal osteotomies affect global postoperative alignment. Adv Orthop 2011:415946, 2011

9. Lafage V, Ames C, Schwab F, Klineberg E, Akbarnia B, Smith J, et al: Changes in thoracic kyphosis negatively impact sagittal alignment after lumbar pedicle subtraction osteotomy: a comprehensive radiographic analysis. Spine (Phila Pa 1976) 37:E180-E187, 2012

10. Lafage V, Schwab F, Skalli W, Hawkinson N, Gagey PM, Ondra S, et al: Standing balance and sagittal plane spinal deformity: analysis of spinopelvic and gravity line parameters. Spine (Phila Pa 1976) 33:1572-1578, 2008

11. Mac-Thiong JM, Transfeldt EE, Mehbod AA, Perra JH, Denis F, Garvey TA, et al: Can c7 plumbline and gravity line predict health related quality of life in adult scoliosis? Spine (Phila Pa 1976) 34:E519-E527, 2009

12. Ryan D, Protopsaltis T, Terran J, Ames C, Li C, Smith J, et al: You scratch my neck, I'll scratch your back: towards a total disability assessment model. Presented at the 41st Annual Cervical Spine Research Society Meeting, Los Angeles, California, 2013 (Poster) (http://www.csrs.org/you-scratchmy-neck-ill-scratch-your-back-towards-a-total-disabilityassessment-model/) [Accessed March 25, 2015]

13. Schwab F, Patel A, Ungar B, Farcy JP, Lafage V: Adult spinal deformity-postoperative standing imbalance: how much can you tolerate? An overview of key parameters in assessing alignment and planning corrective surgery. Spine (Phila Pa 1976) 35:2224-2231, 2010

14. Smith JS, Shaffrey CI, Glassman SD, Carreon LY, Schwab FJ, Lafage V, et al: Clinical and radiographic parameters that distinguish between the best and worst outcomes of scoliosis surgery for adults. Eur Spine J 22:402-410, 2013

15. Smith JS, Shaffrey CI, Lafage V, Blondel B, Schwab F, Hostin R, et al: Spontaneous improvement of cervical alignment after correction of global sagittal balance following pedicle subtraction osteotomy. J Neurosurg Spine 17:300-307, 2012

16. Tang JA, Scheer JK, Smith JS, Deviren V, Bess S, Hart RA, et al: The impact of standing regional cervical sagittal alignment on outcomes in posterior cervical fusion surgery. Neurosurgery 71:662-669, 2012

17. Villavicencio AT, Babuska JM, Ashton A, Busch E, Roeca C, Nelson EL, et al: Prospective, randomized, double-blind clinical study evaluating the correlation of clinical outcomes and cervical sagittal alignment. Neurosurgery 68:1309-1316, 2011

\section{Author Contributions}

Conception and design: Protopsaltis, Ames. Acquisition of data: Scheer, Lafage. Analysis and interpretation of data: Protopsaltis, Scheer, Smith, Lafage. Drafting the article: Protopsaltis, Scheer. Critically revising the article: Protopsaltis, Scheer, Smith, Lafage, Ames. Reviewed submitted version of manuscript: all authors. Approved the final version of the manuscript on behalf of all authors: Protopsaltis. Statistical analysis: Scheer. Administrative/ technical/material support: Lafage. Study supervision: Ames.

\section{Correspondence}

Themistocles S. Protopsaltis, NYU Hospital for Joint Diseases, 301 E. 17th St., Rm. 413, New York, NY 10003. email: tprotopsaltis@gmail.com. 